\title{
A SUBHARMONIC FUNCTION RELATED TO THEOREMS OF BARRY
}

\author{
By Hideharu UEDA
}

Introduction. Let $u(z)$ be a nonconstant subharmonic function in the finite plane and write

$$
m^{*}(r, u)=\inf _{|z|=r} u(z), \quad M(r, u)=\max _{|z|=r} u(z) .
$$

The order and lower order of $u(z), \rho(u)$ and $\mu(u)$ respectively, are by definition

$$
\rho(u)=\lim _{r \rightarrow \infty} \sup \frac{\log M(r, u)}{\log r}, \quad \mu(u)=\liminf _{r \rightarrow \infty} \frac{\log M(r, u)}{\log r} .
$$

If $E$ is a Lebesgue measurable set on the positive real axis, we use the notation $E_{r}=E \cap[1, r]$, and define the upper logarithmic and lower logarithmic densities, respectively, of $E$ by

$$
\begin{aligned}
& \widehat{\log \text { dens }} E=\limsup _{r \rightarrow \infty} \frac{1}{\log r} \int_{E_{r}} t^{-1} d t, \\
& \underline{\log \text { dens }} E=\liminf _{r \rightarrow \infty} \frac{1}{\log r} \int_{E_{r}} t^{-1} d t .
\end{aligned}
$$

Kjellberg [5] proved that, if $0<\mu(u)<1$,

$$
\limsup _{r \rightarrow \infty}-\frac{m^{*}(r, u)}{M(r, u)} \geqq \cos \pi \mu(u) .
$$

Barry showed that, if $0 \leqq \rho(u)<\alpha<1$,

$$
\underline{\log \text { dens }}\left\{r ; m^{*}(r, u)>\cos \pi \alpha M(r, u)\right\} \geqq 1-\rho(u) / \alpha,
$$

and that, if $0 \leqq \mu(u)<\alpha<1$,

$$
\overline{\log \operatorname{dens}}\left\{r ; m^{*}(r, u)>\cos \pi \alpha M(r, u)\right\} \geqq 1-\mu(u) / \alpha \text {. }
$$

(For (1) see [1]; for (2) see [2]). The above estimates (1) and (2) are both sharp in the sense that the sign $\geqq$ cannot be replaced by $>$. In fact, the following result was proved by Hayman [3].

Received January 25, 1983 
THEOREM A. Given any numbers $\rho, \alpha$ such that $0<\rho<\alpha<1$, there exists a subharmonic function $u(z)$ in the finite plane satisfying the following conditions:

( i ) $\rho(u)=\mu(u)=\rho$,

(ii) $\underline{\log \text { dens }} E=\overline{\log \operatorname{dens}} E=1-\rho / \alpha$, where $E$ is defined by

$$
E=\left\{r ; m^{*}(r, u)>\cos \pi \alpha M(r, u)\right\} .
$$

In $\S 1$, we show that the relation $(1)((2))$ is the only essential restriction imposed on $\log \operatorname{dens} E(\overline{\log \operatorname{dens}} E)$, where $E$ is the set defined by (3).

THEOREM 1. Given any numbers $\rho, \alpha, \gamma$ such that $0<\rho<\alpha<1$ and $0 \leqq \gamma \leqq 1$ there exists a subharmonic function $u(z)$ in the finite plane satisfying the following conditions :

(i) $\rho(u)=\mu(u)=\rho$,

(ii) $\log$ dens $E=\overline{\log \operatorname{dens}} E=1-\gamma \rho / \alpha$, where $E$ is defined by (3).

Since Hayman has given examples for $\gamma=1$, we may consider the cases $0 \leqq$ $\gamma<1$. In $\S 1$, we suppose $\gamma>0$. The remaining case $-\gamma=0-$ is handled by minor technical variations of our arguments, and we will omit the proof.

In [6], we showed the following result complementing Theorem A.

THEOREM B. Given any numbers $\mu, \rho$, $\alpha$ such that $0 \leqq \mu<\rho<\alpha<1$, there exists a subharmonic function $u(z)$ in the finite plane satrsfying the following conditions:

(i) $\rho(u)=\rho$,

(ii) $\mu(u)=\mu$,

(iii) $\log$ dens $E=1-\rho / \alpha$,

(iv) $\overparen{\log \operatorname{dens}} E=1-\mu / \alpha$,

where $E$ is the set defined by (3).

Now, it is natural to ask whether only the relations (1) and (2) are essential restrictions imposed on $\underline{\log \text { dens }} E$ and $\overline{\log \text { dens }} E$. I do not know the answer. In $\S 2$, we give examples in this direction.

THEOREM 2. Let $\mu, \rho, \alpha, \beta$ be any numbers such that $0<\mu<\rho<\alpha<1$ and $\beta$ $>\alpha$. If $\beta \leqq 1$, let $\lambda$ be a number satisfying 


$$
\frac{\rho}{\mu} \leqq \lambda<\frac{\beta(\rho-\mu)+(\alpha-\rho) \mu}{\mu(\alpha-\mu)} .
$$

If $\beta>1$, let $\lambda$ be a number satısfying

$$
\frac{\beta(\rho-\mu)+(1-\rho) \mu}{\mu(1-\mu)}<\lambda<\frac{\beta(\rho-\mu)+(\alpha-\rho) \mu}{\mu(\alpha-\mu)} .
$$

Then there exists a subharmonic function $u(z)$ in the finite plane satısfying the following conditions:

(i ) $\rho(u)=\rho$,

(ii) $\mu(u)=\mu$,

(iii) $\overline{\log \text { dens }} E=1-\mu / \beta$,

(iv) $\log \operatorname{dens} E=1-\lambda \mu / \beta$,

where $E$ is defined by (3).

\section{$\S 1$. Proof of Theorem 1 .}

1. Let $\left\{\alpha_{m}\right\}_{0}^{\infty}$ be a decreasing sequence tending to $\alpha$ such that $\alpha_{0}<1$, and set

$$
\beta_{m}=\alpha_{m} \rho(1-\gamma) /\left(\alpha_{m}-\gamma \rho\right) \quad(m=0,1,2, \cdots) .
$$

Define a sequence $\left\{r_{m}\right\}_{0}^{\infty}$ by

$$
r_{0}=1, \quad K_{m} \equiv r_{m+1} / r_{m}=4+m \quad(m \geqq 0) .
$$

Further let $r_{m}^{\prime}$ be the number satisfying

$$
\left(\frac{r_{m}^{\prime}}{r_{m}}\right)^{\alpha}=\left(\frac{r_{m+1}}{r_{m}}\right)^{r \rho} \quad(m=0,1,2, \cdots) .
$$

Then, since $\gamma \rho<\rho<\alpha<\alpha_{m}$, we deduce from (1.2) and (1.3) that

$$
r_{m}<r_{m}^{\prime}<r_{m+1} \quad(m=0,1,2, \cdots) .
$$

Now, we put $\nu(t)$ as follows :

$$
\left\{\begin{array}{l}
\nu(t)=0 \quad(0 \leqq t<1), \quad \nu\left(r_{m}\right)=r_{m}^{o} \quad(m=0,1,2, \cdots), \\
\nu(t) / t^{\alpha}=r_{m}^{\rho-\alpha_{m}} \quad\left(r_{m}<t \leqq r_{m}^{\prime}\right), \quad \nu(t) / t^{\beta_{m}}=r_{m+1}^{\rho-\beta m} \quad\left(r_{m}^{\prime}<t<r_{m+1}\right)
\end{array}\right.
$$

It is easy to see from $(1.1)-(1.4)$ that $\nu(t)(t \geqq 1)$ is a continuous increasing function.

LEMMA 1. $\nu(t)$ has order and lower order equal to $\rho$.

Proof. Assume that $r_{m} \leqq t<r_{m+1}$. From (1.4) and (1.2) we have 


$$
\nu(t) \leqq r_{m+1}^{o} \leqq t^{\rho}\left(\frac{r_{m+1}}{r_{m}}\right)^{\rho}=t^{\rho}(4+m)^{\rho}
$$

and

$$
\nu(t) \geqq r_{m}^{\rho} \geqq t^{\rho}\left(\frac{r_{m}}{r_{m+1}}\right)^{\rho} \geqq t^{\rho}(4+m)^{-\rho} .
$$

By (1.2), we have for $m \geqq 1$

$$
r_{m}=(3+m)(2+m) \cdots 4 \geqq m !=\Gamma(m+1) \sim \sqrt{2 \pi}(m+1)^{m+1 / 2} e^{-m-1} \quad(m \rightarrow \infty),
$$

so that

$$
r_{m} \geqq(m / e)^{m} \quad(m \text { : large enough }) .
$$

Hence, for all sufficiently large $t$

$$
t^{\rho}(4+\log t)^{-\rho}<\nu(t)<t^{\rho}(4+\log t)^{\rho} .
$$

This proves Lemma 1.

2. Put

$$
K_{m}=\left(\log K_{m}^{\prime}\right)^{2 / \rho} .
$$

In view of (1.2), (1.3) and (2.1), we have $r_{m}^{\prime} / K_{m}>K_{m} r_{m}$ and $r_{m+1} / K_{m}>K_{m} r_{m}^{\prime}$ $\left(m \geqq m_{0}\right)$. Now, we define $F_{1}$ and $F_{2}$ as follows:

$$
F_{1}=\bigcup_{m=m_{0}}^{\infty}\left[K_{m} r_{m}, r_{m}^{\prime} / K_{m}\right], \quad F_{2}=\bigcup_{m=m_{0}}^{\infty}\left[K_{m} r_{m}^{\prime}, r_{m+1} / K_{m}\right] .
$$

Then we have the following

Lemma 2. $\log \operatorname{dens} F_{1}=\gamma \rho / \alpha, \quad \log \operatorname{dens} F_{2}=1-\gamma \rho / \alpha$.

Proof. Let $R$ be a large positive number and let $m_{1}$ be the integer such that $r_{m_{1}}^{\prime} / K_{m_{1}} \leqq R<r_{m_{1}+1}^{\prime} / K_{m_{1}+1}$. Suppose first that $r_{m_{1}}^{\prime} / K_{m_{1}} \leqq R<K_{m_{1}+1} r_{m_{1}+1}$ $\left(m_{1} \geqq m_{0}\right)$. Then we have from (2.2), (1.2) and (1.3) that

$$
\begin{aligned}
\int_{\left(F_{1}\right)_{R}} \frac{d t}{t} & =\sum_{m=m_{0}}^{m_{1}} \int_{K_{m} r_{m}}^{r_{m}^{\prime} / K_{m}} \frac{d t}{t} \\
& =\sum_{m=m_{0}}^{m_{1}}\left\{\log \left(\frac{r_{m}^{\prime}}{r_{m}}\right)-2 \log K_{m}\right\}=\sum_{m=m_{0}}^{m_{1}}\left\{\frac{\gamma \rho}{\alpha_{m}} \log K_{m}^{\prime}-2 \log K_{m}\right\} .
\end{aligned}
$$

In view of $(2.2)$

$$
\log K_{m}=o\left(\log K_{m}^{\prime}\right) \quad(m \rightarrow \infty) .
$$

Also $\alpha_{m} \downarrow \alpha(m \rightarrow \infty)$. Hence given $\varepsilon>0$, we can choose $N=N(\varepsilon)$, so that for $m_{1} \geqq N$ 


$$
\begin{aligned}
\frac{\gamma \rho}{\alpha} \log \frac{r_{m_{1}+1}}{r_{m_{0}}} & >\int_{\left(F_{1}\right)_{R}} \frac{d t}{t} \\
& >\frac{\gamma \rho}{\alpha}(1-\varepsilon) \sum_{m=N}^{m_{1}} \log K_{m}^{\prime}=\frac{\gamma \rho}{\alpha}(1-\varepsilon) \log \frac{r_{m_{1}+1}}{r_{N}} .
\end{aligned}
$$

Since $R \in\left[r_{m_{1}}^{\prime} / K_{m_{1}}, K_{m_{1}+1} r_{m_{1}+1}\right]$, we have

$$
\begin{aligned}
\frac{(\gamma \rho / \alpha)\left(\log r_{m_{1}+1}-\log r_{m_{0}}\right)}{\log r_{m_{1}}^{\prime}-\log K_{m_{1}}} & >\frac{1}{\log R} \int_{\left(F_{1}\right)_{R}} \frac{d t}{t} \\
& >\frac{(\gamma \rho / \alpha)(1-\varepsilon)\left(\log r_{m_{1}+1}-\log r_{N}\right)}{\log K_{m_{1}+1}+\log r_{m_{1}+1}} .
\end{aligned}
$$

By (1.2), (1.3)

$$
\log K_{m}^{\prime}=o\left(\log r_{m}\right) \quad(m \rightarrow \infty) .
$$

Using (2.3) and (2.5), we deduce from (2.4) that

$$
\frac{\gamma \rho}{\alpha}(1+\varepsilon)>\frac{1}{\log R}-\int_{\left(F_{1}\right)_{R}} \frac{d t}{t}>\frac{\gamma \rho}{\alpha}(1-\varepsilon)^{2}
$$

for all sufficiently large $R \in \bigcup_{m \geqq m_{0}}\left[r_{m}^{\prime} / K_{m}, K_{m+1} r_{m+1}\right]$.

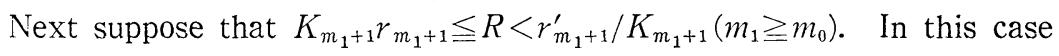

$$
\int_{\left(F_{1}\right)_{R}} \frac{d t}{t}=\sum_{m=m_{0}}^{m_{1}}\left\{\frac{\gamma \rho}{\alpha_{m}} \log K_{m}^{\prime}-2 \log K_{m}\right\}+\log \frac{R}{K_{m_{1}+1} r_{m_{1}+1}} .
$$

Since $R<r_{m_{1}+1}^{\prime} / K_{m_{1}+1}$, we have

$$
\begin{aligned}
\frac{1}{\log R} \int_{\left(F_{1}\right)_{R}} \frac{d t}{t} & <\frac{(\gamma \rho / \alpha) \log \left(r_{m_{1}+1} / r_{m_{0}}\right)+\log R-\log r_{m_{1}+1}}{\log R} \\
& <1-\frac{(1-\gamma \rho / \alpha) \log r_{m_{1}+1}}{\log R}<1-\frac{(1-\gamma \rho / \alpha) \log r_{m_{1}+1}}{\log r_{m_{1+1}}^{\prime}-\log K_{m_{1+1}}} .
\end{aligned}
$$

On the other hand, since $R>K_{m_{1}+1} r_{m_{1}+1}$

$$
\begin{aligned}
\frac{1}{\log R} \int_{\left(F_{1}\right)_{R}} \frac{d t}{t}>-\frac{(\gamma \rho / \alpha)(1-\varepsilon) \log \left(r_{m_{1}+1} / r_{N}\right)+\log \left(R / K_{m_{1}+1} r_{m_{1}+1}\right)}{\log R} \\
\quad=\frac{\log R-\log K_{m_{1}+1}-\{1-(1-\varepsilon)(\gamma \rho / \alpha)\} \log r_{m_{1}+1}-(1-\varepsilon)(\gamma \rho / \alpha) \log r_{N}}{\log R} \\
>1-o(1)-\{1-(1-\varepsilon) \gamma \rho / \alpha\} \frac{\log r_{m_{1+1}}}{\log K_{m_{1}+1} r_{m_{1}+1}}\left(m_{1} \rightarrow \infty\right) .
\end{aligned}
$$

Thus for all sufficiently large $R \in \bigcup_{m \geqq m_{0}}\left[K_{m+1} r_{m+1}, r_{m+1} / K_{m+1}\right]$

$$
\frac{\gamma \rho}{\alpha}(1-\varepsilon)^{2}<\frac{1}{\log R} \int_{\left(F_{1}\right)_{R}} \frac{d t}{t}<\frac{\gamma \rho}{\alpha}+\varepsilon(1-\gamma \rho / \alpha) .
$$


Combining (2.6) and (2.7), we have

$$
\frac{\gamma \rho}{\alpha}(1-\varepsilon)^{2}<\frac{1}{\log R} \int_{\left(F_{1}\right)_{R}} \frac{d t}{t}<\frac{\gamma \rho}{\alpha}+\varepsilon
$$

for all sufficiently large $R$. Hence

$$
\frac{\gamma \rho}{\alpha}(1-\varepsilon)^{2} \leqq \underline{\log \operatorname{dens}} F_{1} \leqq \overline{\log \operatorname{den} \mathrm{s}} F_{1} \leqq \frac{\gamma \rho}{\alpha}+\varepsilon .
$$

Since $\varepsilon$ is an arbitrary positive number, we deduce that

$$
\log \operatorname{dens} F_{1}=\gamma \rho / \alpha \text {. }
$$

The proof of $\log$ dens $F_{2}=1-\gamma \rho / \alpha$ is quite similar to the above one.

3. Define $\nu(t)(t \geqq 0)$ by (1.4). It follows from Lemma 1 that

$$
\int_{1}^{\infty} \frac{d \nu(t)}{t}=-1+\int_{1}^{\infty} \frac{\nu(t)}{t^{2}} d t<\infty
$$

Hence

$$
u(z) \equiv \int_{0}^{\infty} \log \left|1+\frac{z}{t}\right| d \nu(t)=\operatorname{Re}\left\{\int_{1}^{\infty} \frac{z \nu(t)}{t(t+z)} d t\right\}
$$

is subharmonic in the finite plane (See [4, Theorems 4.1 and 4.2].). Clearly

$$
M(r, u)=r \int_{1}^{\infty} \frac{\nu(t)}{t(t+r)} d t
$$

By Lemma 1 and (3.2), $\rho(u)=\mu(u)=\rho$.

Lemma 3. Suppose that $z=r e^{i \theta}$, Then if $u(z)$ is defined by (3.1), we have the following estimates.

$$
\begin{array}{r}
\left|u(z)-\frac{\pi \nu(r)}{\sin \pi \alpha_{m}} \cos \alpha_{m} \theta\right|<O\left(\left(\frac{\log K_{m}^{\prime}}{K_{m}^{\rho}}+\frac{1}{K_{m}^{1-\alpha}-\overline{\alpha_{0}}}\right) \nu(r)\right) \\
\left(K_{m} r_{m} \leqq r \leqq r_{m}^{\prime} / K_{m}\right), \\
\left|u(z)-\frac{\pi \nu(r)}{\sin \pi \beta_{m}} \cos \beta_{m} \theta\right|<O\left(\left(\frac{\log K_{m}^{\prime}}{K_{m}^{\rho}}+\frac{1}{K_{m}^{1-\beta}}\right) \nu(r)\right) \\
\left(K_{m} r_{m}^{\prime} \leqq r \leqq r_{m+1} / K_{m}\right),
\end{array}
$$

where $\beta=\lim _{m \rightarrow \infty} \beta_{m}=\alpha \rho(1-\gamma) /(\alpha-\gamma \rho)$.

Proof. We prove only (3.3). By (3.1) 


$$
\begin{aligned}
u(z) & =\operatorname{Re}\left\{z \int_{1}^{\infty} \frac{\nu(t)}{t(t+z)} d t\right\}=\operatorname{Re}\left\{z \int_{1}^{r_{m}} \frac{\nu(t)}{t(t+z)} d t\right\} \\
& +\operatorname{Re}\left\{z \int_{r_{m}}^{r_{m^{\prime}}} \frac{\nu(t)}{t(t+z)} d t\right\}+\operatorname{Re}\left\{z \int_{r_{m^{\prime}}}^{\infty} \frac{\nu(t)}{t(t+z)} d t\right\} \equiv I_{1}+I_{2}+I_{3}, \quad \text { say . }
\end{aligned}
$$

For $t \leqq r_{m}$, we have $|z /(t+z)| \leqq r /\left(r-r_{m}\right) \leqq K_{m} /\left(K_{m}-1\right) \leqq 2$, so that

$$
\begin{aligned}
\left|I_{1}(z)\right| & \leqq 2 \int_{1}^{r_{m}} \frac{\nu(t)}{t} d t=2 \sum_{\mu=1}^{m} \int_{r_{\mu-1}}^{r_{\mu}} \frac{\nu(t)}{t} d t \leqq 2 \sum_{\mu=1}^{m} \nu\left(r_{\mu}\right) \log K_{\mu-1}^{\prime} \\
& \leqq 2 \log K_{m-1}^{\prime} \sum_{\mu=1}^{m} r_{\mu}^{\rho} \leqq 2\left(\log K_{m-1}^{\prime}\right) r_{m}^{\rho} \sum_{\mu=0}^{\infty} 4^{-\mu \rho}=2 r_{m}^{\rho}\left(\log K_{m-1}^{\prime}\right) \frac{1}{1-4^{-\rho}} \\
& <\frac{8}{\rho} r_{m}^{o}\left(\log K_{m-1}^{\prime}\right)=\frac{8}{\rho}\left(r_{m} / r\right)^{\alpha} m \nu(r)\left(\log K_{m-1}^{\prime}\right) \\
& \leqq \frac{8}{\rho} K_{m}^{-\alpha} \mathcal{\nu}(r)\left(\log K_{m-1}^{\prime}\right)<\frac{8}{\rho} K_{m}^{-\rho} \nu(r)\left(\log K_{m-1}^{\prime}\right)
\end{aligned}
$$

Assume next that $r_{m} \leqq t \leqq r_{m}^{\prime}$. Then, we have $\nu(t)=r_{m}^{-\alpha-\alpha} t^{\alpha}$. Hence from Lemma 1 in [3] we have for $|\theta|<\pi$

$$
\left|I_{2}(z)-\frac{\pi \nu(r)}{\sin \pi \alpha_{m}} \cos \alpha_{m} \theta\right|<\left\{\frac{2}{\alpha_{m} K_{m}^{\alpha_{m}}}+\frac{2}{\left(1-\alpha_{m}\right) K_{m}^{1-\alpha_{m}}}\right\} \nu(r) .
$$

Finally for $t \geqq r_{m}^{\prime},|(t+z) / t| \geqq\left(r_{m}^{\prime}-r\right) / r_{m}^{\prime} \geqq 1-1 / K_{m} \geqq 1 / 2$, so that

$$
\left|I_{3}(z)\right|<2 r \int_{r_{m}^{\prime}}^{\infty} \nu(t) / t^{2} d t
$$

Since $\nu(t) / t^{\alpha_{0}}$ decreases for all $t$, we deduce that

$$
\int_{r_{m}^{\prime}}^{\infty} \frac{\nu(t)}{t^{2}} d t \leqq \int_{r_{m}^{\prime}}^{\infty} \frac{\nu(r) t^{\alpha_{0}}}{r^{\alpha_{0}}}-\frac{1}{t^{2}} d t=\frac{\nu(r)}{r^{\alpha_{0}}\left(1-\alpha_{0}\right)\left(r_{m}^{\prime}\right)^{1-\alpha_{0}}} .
$$

Hence

$$
\left|I_{3}(z)\right| \leqq \frac{2 \nu(r)}{1-\alpha_{0}}\left(\frac{r}{r_{m}^{\prime}}\right)^{1-\alpha_{0}} \leqq \frac{2 K_{m}^{\alpha_{0}-1}}{1-\alpha_{0}} \nu(r)
$$

Combining (3.5)-(3.8), we obtain (3.3) for $|\theta|<\pi$.

Now, we consider the case $|\theta|=\pi$. Since $\log \left|1+\frac{r e^{i \theta}}{t}\right|$ is a decreasing function of $\theta$ in $(0, \pi)$, and since $\log \left|1+\frac{r e^{i \theta}}{t}\right| \leqq \log \left(1+\frac{r}{t}\right)(0<\theta \leqq \pi)$ with $\int_{0}^{\infty} \log \left(1+\frac{r}{t}\right) d \nu(t)<\infty$, the monotone convergence theorem shows that $\lim _{\theta \rightarrow \pi-} u\left(r e^{2 \theta}\right)$ $=\lim _{\theta \rightarrow-\pi+} u\left(r e^{i \theta}\right)=u(-r)$. Hence (3.3) is valid also for $|\theta|=\pi$. 
Assume now that $K_{m} r_{m} \leqq r \leqq r_{m}^{\prime} / K_{m}\left(m \geqq m_{0}\right)$. We deduce from Lemma 3 that

$$
M(r, u)=\left\{\frac{\pi}{\sin \pi \alpha_{m}}+O\left(\frac{\log K_{m}^{\prime}}{K_{m}^{\rho}}+\frac{1}{K_{m}^{1-\alpha_{0}}}\right)\right\} \nu(r),
$$

and that

$$
m^{*}(r, u)=\left\{\frac{\pi \cos \pi \alpha_{m}}{\sin \pi \alpha_{m}}-O\left(\frac{\log K_{m}^{\prime}}{K_{m}^{o}}+\frac{1}{K_{m}^{1-\alpha_{0}}}\right)\right\} \nu(r)
$$

Here we choose $\alpha_{m}=\alpha+\frac{1-\alpha}{2}\left(1+\log ^{+} \log ^{+} m\right)^{-1}$. Then

$$
\begin{aligned}
\frac{m^{*}(r, u)}{M(r, u)} & \leqq \cos \pi \alpha_{m}+O\left(\frac{1}{\log m}+\frac{1}{(\log m)^{2\left(1-\alpha_{0}\right) / \rho}}\right)+O\left(\frac{\log r}{\nu(r)}\right) \\
& \leqq \cos \pi \alpha_{m}+O\left(\frac{1}{\log m}+\frac{1}{(\log m)^{2\left(1-\alpha_{0} / \rho\right)}}\right)<\cos \pi \alpha_{m}+o\left(\alpha_{m}-\alpha\right)<\cos \pi \alpha
\end{aligned}
$$

Hence for all sufficiently large $r \in F_{1}$

$$
m^{*}(r, u)<\cos \pi \alpha M(r, u) .
$$

Next assume that $K_{m} r_{m}^{\prime} \leqq r \leqq r_{m+1} / K_{m}\left(m \geqq m_{0}\right)$. By (1.1) and the definition of $\beta, \alpha_{m}-\alpha=O\left(\beta-\beta_{m}\right)(m \rightarrow \infty)$. Hence

$$
\begin{aligned}
\frac{m^{*}(r, u)}{M(r, u)} & \geqq \cos \pi \beta_{m}-O\left(\frac{1}{\log m}+\frac{1}{(\log m)^{2(1-\beta) / \rho}}\right)-O\left(\frac{\log r}{\nu(r)}\right) \\
& \geqq \cos \pi \beta_{m}-o\left(\alpha_{m}-\alpha\right) \geqq \cos \pi \beta_{m}-o\left(\beta-\beta_{m}\right)>\cos \pi \beta .
\end{aligned}
$$

Thus for all sufficiently large $r \in F_{2}$

$$
m^{*}(r, u)>\cos \pi \beta M(r, u)>\cos \pi \rho M(r, u)>\cos \pi \alpha M(r, u) .
$$

4. Define $E$ by (3). Then by (3.9) and (3.10)

$$
F_{2} \cap\left[R_{0}, \infty\right) \subset E \subset[1, \infty) \backslash F_{1} \cap\left[R_{0}, \infty\right)
$$

for a large positive constant $R_{0}$. Hence

$$
\underline{\log \operatorname{dens}} F_{2} \leqq \underline{\log \text { dens }} E \leqq \overline{\log \operatorname{dens}} E \leqq 1-\underline{\log \operatorname{dens}} F_{1} .
$$

From this and Lemma 2 we deduce that $\log$ dens $E=1-\gamma \rho / \alpha$. This completes the proof of Theorem 1 .

\section{$\S 2$. Proof of Theorem 2 .}

5. Put

$$
\gamma=\frac{(\beta-\mu \lambda)(\rho-\mu)}{(\lambda-1) \mu}+\rho
$$


and

$$
\delta=\frac{\lambda \mu-\rho}{\lambda-1}
$$

The choice of $\beta$ and $\lambda$ implies $\alpha<\gamma<1$ and $0 \leqq \delta<\mu$. Define two sequences $\left\{r_{m}\right\}_{0}^{\infty},\left\{r_{m}^{\prime}\right\}_{1}^{\infty}$ as follows :

$$
\begin{gathered}
r_{0}=1, \quad r_{1}=3, \quad r_{m+1}=r_{m}^{\lambda(\beta-\mu) /(\beta-\mu \lambda)} \quad(m=1,2, \cdots), \\
r_{m}^{\prime}=r_{m}^{(\beta-\mu) /(\beta-\mu \lambda)} \quad(m=1,2, \cdots) .
\end{gathered}
$$

It is easy to see that $r_{m}<r_{m}^{\prime}<r_{m+1} \quad(m=1,2, \cdots)$.

Now, we define $\nu(t)$ so that

$$
\left\{\begin{array}{l}
\nu(t)=0 \quad(0 \leqq t<1), \quad \nu\left(r_{m}\right)=r_{m}^{\mu} \quad(m=0,1,2, \cdots), \\
\nu(t)=r_{m}^{\mu-\gamma} t^{\gamma} \quad\left(r_{m} \leqq t \leqq r_{m}^{\prime} ; m \geqq 1\right), \quad \nu(t)=r_{m+1}^{\mu-\delta} \delta^{\delta} \quad\left(r_{m}^{\prime} \leqq t<r_{m+1} ; m \geqq 1\right) .
\end{array}\right.
$$

We deduce from $(5.1)-(5.5)$ that $\nu(t)(t \geqq 1)$ is a continuous increasing function.

LEMMA 4. $\nu(t)$ has order $\rho$ and lower order $\mu$.

Proof. We note that $\nu\left(r_{m}\right)=r_{m}^{\mu}$ and $\nu\left(r_{m}^{\prime}\right)=\left(r_{m}^{\prime}\right)^{\rho}(m=1,2, \cdots)$. Hence it suffices to show that $t^{\mu} \leqq \nu(t) \leqq t^{\rho}\left(r_{m} \leqq t<r_{m+1} ; m=1,2, \cdots\right)$. Assume first that $r_{m} \leqq t \leqq r_{m}^{\prime}$. From (5.5) we have $\nu(t) / t^{\mu}=\left(t / r_{m}\right)^{\gamma-\mu} \geqq 1$. On the other hand, we deduce from (5.5), (5.4) and (5.1) that $\nu(t) / t^{\rho}=\gamma_{m}^{\mu-\gamma} t^{\gamma-\rho} \leqq r_{m}^{\mu-\gamma}\left(r_{m}^{\prime}\right)^{\gamma-\rho}=\gamma_{m}^{\mu-\gamma+(\beta-\mu)(\gamma-\rho) / \beta-\mu \lambda}$ $=r_{m}^{0}=1$. Assume next that $r_{m}^{\prime} \leqq t<r_{m+1}$. By $(5.5) \nu(t) / t^{\mu}=\left(r_{m+1} / t\right)^{\mu-\delta} \geqq 1$, and from $(5.2)-(5.5)$ we have

$$
\nu(t) / t^{\rho}=r_{m+1}^{\mu-\delta} t^{\delta-\rho} \leqq r_{m+1}^{\mu-\delta}\left(r_{m}^{\prime}\right)^{\delta-\rho}=r_{m}^{(\mu-\delta) \lambda(\beta-\mu) /(\beta-\mu \lambda)+(\beta-\mu)(\delta-\rho) /(\beta-\mu \lambda)}=r_{m}^{0}=1 .
$$

6. We set $K_{m}^{\prime}=r_{m+1} / r_{m}(m=1,2, \cdots)$ and define

$$
K_{m}=\left(\log K_{m}^{\prime}\right)^{2 / \mu} \text {. }
$$

In view of (5.3), (5.4) and (6.1), we have $r_{m}^{\prime} / K_{m}>K_{m} r_{m}$ and $r_{m+1} / K_{m}>K_{m} r_{m}^{\prime}$ $\left(m \geqq m_{0}\right)$. Define two sets $F_{1}$ and $F_{2}$ by $(2.2)$. Then the same reasoning as in the proof of Lemma 2 gives the following.

LEMMA 5. $\underline{\log \operatorname{dens}} F_{1}=\mu / \beta, \quad \overline{\log \operatorname{dens}} F_{1}=\lambda \mu / \beta, \quad \underline{\log \operatorname{dens}} F_{2}=1-\lambda \mu / \beta$, $\overline{\log \operatorname{dens}} F_{2}=1-\mu / \beta$.

7. Define $\nu(t)$ by (5.5). Then from Lemma 4 we deduce that

$$
u(z) \equiv \int_{0}^{\infty} \log \left|1+\frac{z}{t}\right| d \nu(t)=\operatorname{Re}\left\{\int_{1}^{\infty} \frac{z \nu(t)}{t(t+z)} d t\right\}
$$

is subharmonic in the finite plane. Clearly 


$$
M(r, u)=r \int_{1}^{\infty} \frac{\nu(t)}{t(t+r)} d t
$$

Here we prove the following

LEMMA 6. $\rho(u)=\rho, \quad \mu(u)=\mu$.

Proof. As noted in the proof of Lemma 4, $t^{\mu} \leqq \nu(t) \leqq t^{\rho}(t \geqq 1)$. From this and (7.2) it is easy to see that $\mu \leqq \mu(u) \leqq \rho(u) \leqq \rho$. We proceed to show that $\rho(u) \geqq \rho$. For this purpose, note that $N(r)=\int_{1}^{r} \nu(t) t^{-1} d t$ has the same order as $\nu(r)$, and so by Lemma 4 it has order $\rho$. Further by the subharmonic from of Jensen's Theorem

$$
M(r, u) \geqq \frac{1}{2 \pi} \int_{-\pi}^{\pi} u\left(r e^{i \theta}\right) d \theta=N(r) .
$$

Thus we have $\rho(u) \geqq \rho$. It remains to prove that $\mu(u) \leqq \mu$. By $(7.2)$

$$
M\left(r_{m}, u\right)=r_{m}\left(\int_{1}^{r_{m}}+\int_{r_{m}}^{\infty}\right) \frac{\nu(t)}{t\left(t+r_{m}\right)} d t \equiv J_{1}+J_{2}, \quad \text { say . }
$$

Clearly

$$
J_{1} \leqq \int_{1}^{r_{m}} \frac{\nu(t)}{t} d t
$$

Computing as in (3.6), we have

$$
J_{1} \leqq \frac{4}{\mu} \gamma_{m}^{\mu} \log K_{m-1}^{\prime}=\frac{4(\lambda-1) \beta}{\mu \lambda(\beta-\mu)} r_{m}^{\mu} \log \gamma_{m}
$$

Since $\nu(t) / t^{r}$ decreases for all $t$, we have

$$
\begin{aligned}
J_{2} & \leqq r_{m} \int_{r_{m}}^{\infty} \frac{\nu(t)}{t^{2}} d t \leqq r_{m} \nu\left(r_{m}\right) \frac{1}{r_{m}^{\gamma}} \int_{r_{m}}^{\infty} t^{\gamma-2} d t \\
& =\frac{\nu\left(r_{m}\right)}{1-\gamma}=\frac{r_{m}^{\prime \prime}}{1-\gamma} .
\end{aligned}
$$

Combining (7.3)-(7.5), we obtain

Hence

$$
M\left(r_{m}, u\right) \leqq O\left(r_{m}^{\prime \prime} \log r_{m}\right) \quad(m \rightarrow \infty) .
$$

$$
\mu(u) \leqq \liminf _{m \rightarrow \infty} \frac{\log M\left(r_{m}, u\right)}{\log r_{m}} \leqq \mu
$$

8. We first suppose that $\lambda>\rho / \mu$. By (5.2) $\delta>0$. In this case the same arguments as in the proof of Lemma 3 give 


$$
\begin{array}{r}
\left|u(z)-\frac{\pi \nu(r)}{\sin \pi \gamma} \cos \gamma \theta\right|<O\left(\left(\frac{\log K_{m}^{\prime}}{K_{m}^{\mu}}+\frac{1}{K_{m}^{1-\gamma}}\right) \nu(r)\right) \\
\left(z=r e^{i \theta}, K_{m} r_{m} \leqq r \leqq r_{m}^{\prime} / K_{m}, m \geqq m_{0}\right),
\end{array}
$$

and

$$
\begin{aligned}
&\left|u(z)-\frac{\pi \nu(r)}{\sin \pi \delta} \cos \delta \theta\right|<O\left(\left(\frac{\log K_{m}^{\prime}}{K_{m}^{\mu}}+\frac{1}{K_{m}^{1-\delta}}\right) \nu(r)\right) \\
&\left(z=r e^{i \theta}, K_{m} r_{m}^{\prime} \leqq r \leqq r_{m+1} / K_{m}, m \geqq m_{0}\right)
\end{aligned}
$$

Since $\alpha<\gamma<1$, we deduce from (8.1) that

$$
m^{*}(r, u)<\cos \pi \alpha M(r, u) \quad\left(r \in F_{1}, r \geqq R_{0}\right) .
$$

Similarly, by (8.2) and the fact that $0<\delta<\alpha$

$$
m^{*}(r, u)>\cos \pi \alpha M(r, u) \quad\left(r \in F_{2}, r \geqq R_{0}\right) .
$$

From (8.3) and (8.4) it follows that

$$
F_{2} \cap\left[R_{0}, \infty\right) \subset E \subset[1, \infty) \backslash\left(F_{1} \cap\left[R_{0}, \infty\right)\right)
$$

for a large positive constant $R_{0}$. Hence

$$
\underline{\log \operatorname{dens}} F_{2} \leqq \underline{\log \text { dens }} E \leqq 1-\overline{\log \operatorname{dens}} F_{1},
$$

and

$$
\overline{\log \text { dens }} F_{2} \leqq \overline{\log \operatorname{dens}} E \leqq 1-\underline{\log \text { dens }} F_{1} .
$$

Combining (8.5), (8.6) with Lemma 5, we have

$$
\underline{\log \operatorname{dens}} E=1-\lambda \mu / \beta, \quad \overline{\log \operatorname{dens}} E=1-\mu / \beta \text {. }
$$

Next, we suppose that $\lambda=\rho / \mu$. It is easy to see that (8.1) remains true this case. We estimate $u\left(r e^{i \theta}\right)$ for $K_{m} r_{m}^{\prime} \leqq r \leqq r_{m+1} / K_{m}\left(m \geqq m_{0}\right)$. We write

$$
\begin{aligned}
u(z) & =\operatorname{Re}\left\{\left(\int_{1}^{r_{m}}+\int_{r_{m}}^{r_{m}^{\prime}}+\int_{r_{m}^{\prime}}^{r_{m+1}}+\int_{r_{m+1}}^{\infty}\right) \frac{z \nu(t)}{t(t+z)} d t\right\} \\
& \equiv I_{1}+I_{2}+I_{3}+I_{4}, \text { say. }
\end{aligned}
$$

For $t \leqq r_{m}$,

$$
\left|I_{1}(z)\right| \leqq 2 \int_{1}^{r_{m}} \frac{\nu(t)}{t} d t
$$

Hence by (7.4)

$$
\left|I_{1}(z)\right| \leqq \frac{8(\lambda-1) \beta}{\mu \lambda(\beta-\mu)} r_{m}^{\mu} \log r_{m}=\frac{8(\lambda-1) \beta(\beta-\mu \lambda)}{\mu(\beta-\mu)^{2} \lambda^{2}} r_{m+1}^{(\mu / \lambda)(\beta-\mu \lambda) /(\beta-\mu)} \log r_{m+1} .
$$


Next,

$$
\left|I_{2}(z)\right| \leqq 2 \int_{r_{m}}^{r_{m}^{\prime}} \frac{\nu(t)}{t} d t=2 \int_{r_{m}}^{r_{m}^{\prime}} \gamma_{m}^{\mu-\gamma} t^{\gamma-1} d t<\frac{2}{\gamma} \gamma_{m}^{\mu-\gamma}\left(r_{m}^{\prime}\right)^{\gamma}=\frac{2}{\gamma} r_{m+1}^{\mu}
$$

Since $\nu(t) / t^{r}$ is a decreasing function, we have

$$
\begin{aligned}
\left|I_{4}(z)\right| & \leqq 2 r \int_{r_{m+1}}^{\infty} \frac{\nu(t)}{t^{2}} d t \leqq 2 r \int_{r_{m+1}}^{\infty} r_{m+1}^{\mu-\gamma} t^{\gamma-2} d t \\
& =\frac{2 \gamma \cdot r_{m+1}^{\mu-1}}{1-\gamma} \leqq \frac{2 r_{m+1}^{\mu}}{(1-\gamma) K_{m}}
\end{aligned}
$$

Finally, for $|\arg z|<\pi$

$$
\begin{aligned}
I_{3}(z) & =r_{m+1}^{\mu} \operatorname{Re} \int_{r_{m}^{\prime}}^{r_{m+1}} \frac{z}{t(t+z)} d t=r_{m+1}^{\prime \prime} \log \left|\frac{r_{m+1}}{r_{m+1}+z} \frac{r_{m}^{\prime}+z}{r_{m}^{\prime}}\right| \\
& =r_{m+1}^{\mu}\left\{\log \frac{r}{r_{m}^{\prime}}+\log \left|\frac{1+\left(r_{m}^{\prime} / z\right)}{1+\left(z / r_{m+1}\right)}\right|\right\} .
\end{aligned}
$$

Hence

$$
\left|I_{3}(z)-r_{m+1}^{\mu} \log \frac{r}{r_{m}^{\prime}}\right| \leqq r_{m+1}^{\mu} \log \frac{1+K_{m}^{-1}}{1-K_{m}^{-1}} \leqq \frac{3}{K_{m}} r_{m+1}^{\mu} .
$$

Combining (8.7)-(8.11) we deduce that for $|\theta|<\pi$

$$
\left|u\left(r e^{i \theta}\right)-\left(\log \frac{r}{r_{m}^{\prime}}\right) r_{m+1}^{\mu}\right| \leqq O\left(r_{m+1}^{\prime}\right) \quad\left(K_{m} r_{m}^{\prime} \leqq r \leqq r_{m+1} / K_{m}\right) .
$$

However as noted in 4, $\lim _{\theta \rightarrow \pi_{-}} u\left(r e^{i \theta}\right)=\lim _{\theta \rightarrow-\pi_{+}} u\left(r e^{i \theta}\right)=u(-r)$, so that (8.12) holds also for $|\theta|=\pi$.

Since $\alpha<\gamma<1$, we obtain from (8.1) that

$$
m^{*}(r, u)<\cos \pi \alpha M(r, u) \quad\left(r \in F_{1}, r \geqq R_{0}\right) .
$$

On the other hand, (8.12) gives

$$
m^{*}(r, u)>\cos \pi \alpha M(r, u) \quad\left(r \in F_{2}, r \geqq R_{0}\right) .
$$

Combining (8.13), (8.14) with Lemma 5, we have

$$
\text { log dens } E=1-\lambda \mu / \beta, \quad \overline{\log \operatorname{dens}} E=1-\mu / \beta .
$$

This completes the proof of Theorem 2 . 


\section{REFERENCES}

[1] Barry, P.D., On a theorem of Besicovitch, Quart. J. Math. Oxford (2) 14 (1963), 293-302.

[2] BARRY, P.D., On a theorem of Kjellberg, ibid. (2) 15 (1964), 179-191.

[3] Hayman, W.K., Some examples related to the $\cos \pi \rho$ theorem, Mathematical essays dedicated to A. J. Macintyre Ohio Univ. Press (1970), 149-170.

[4] Hayman, W. K. And Kennedy, P.B., Subharmonic Functions, Vol. 1 Academic Press.

[5] KJellberG, B., On the minımum modulus of entire functions of lower order less than one, Math. Scand. 8 (1960), 189-197.

[6] UEDA, H., An entire function related to theorems of Barry, Koda1 Math. J. 5 No. 2, (1982), 289-302.

Department of Mathematios

Daido Institute of Technology

Daido-cho, Minami-ku, Nagoya, Japan 